\section{Cadmium isotope variations \\ in Neoproterozoic carbonates - A tracer of biologic production?}

\section{S.V. Hohl ${ }^{1,2 *}$, S.J.G. Galer ${ }^{3}$, A. Gamper ${ }^{4}$, H. Becker ${ }^{1}$}

open 2 access

\section{Abstract}

doi: 10.7185/geochemlet.1704

Cadmium concentrations and stable isotopic compositions in seawater are important tools for studying the biogeochemical cycling of $\mathrm{Cd}$ in the modern oceans and as a proxy for micronutrient utilisation by phytoplankton. It is now well established that $\mathrm{Cd}$ isotopes become "heavier" as the primary production in the surface ocean increases, even though the mechanism driving the isotopic fractionation is still debated. Here, we use this property of $\mathrm{Cd}$ isotopes to examine changes that took place in the oceans during the emergence of multicellular life in the Neoproterozoic. Isotopic compositions and concentrations of $\mathrm{Cd}$, $\mathrm{N}$ and $\mathrm{C}$ are reported in shallow-water carbonates of Ediacaran age from the Xiaofenghe section on the Yangtze Platform, South China. The Cd isotope data - reported as $\varepsilon^{112 / 110} \mathrm{Cd}$ - show positive excursions in the cap dolomites, while significantly lighter $\mathrm{Cd}$ is found in the - show positive exursions in the cap dolonites, while significa c

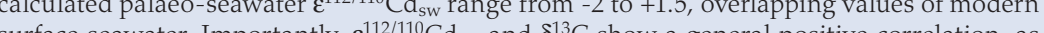
surface seawater. Importantly, $\varepsilon$ $\varepsilon \mathrm{Cd}_{\mathrm{sw}}$ and $\delta{ }^{1} \mathrm{C}$ show a general positive correlation, as would be expected in bio-productive environments. However, the trend to lighter $\varepsilon{ }^{\mathrm{l}} \mathrm{Cd}$ up-section is not that explicitly expected for an "explosion of life" at the end of the Ediacaran. The upper Doushantuo also displays substantial fluctuations in REE abundances, ${ }^{15} \mathrm{~N}$ and $\delta^{13} \mathrm{C}$, which may be due to estuarine mixing. Our data suggest that the variations in $\varepsilon^{112 / 110} \mathrm{Cd}$ are a result of biologically-induced fractionation in at least some of the Ediacaran carbonates at Xiaofenghe. Further $\mathrm{Cd}$ isotope fractionation processes are clearly playing a role as well, such as precipitation of sulphides under anoxic pore-water conditions and fractionation into inorganic carbonates under variable salinity conditions. These effects have to be evaluated carefully when using $\mathrm{Cd}$ isotope systematics in ancient marine carbonates to look for palaeo-productivity signals.

Received 19 May 2016 | Accepted 31 August 2016 | Published 9 September 2016

1. Institut für Geologische Wissenschaften, Freie Universität Berlin, Germany

State Key Laboratory for Mineral Deposits Research, Department of Earth Sciences, Nanjing University Nanjing, China

Corresponding author (email: shohl@zedat.fu-berlin.de)

Max-Planck-Institut für Chemie, Abteilung Klimageochemie, Mainz, Germany

Museum für Naturkunde Berlin, Leibniz Institute for Evolution and Biodiversity Science, Berlin Germany

\title{
Introduction
}

The oceans underwent some of the most remarkable transformations in Earth's history in the Neoproterozoic, such as the appearance of animal life (Knoll, 2015), major excursions in sulphur and carbon isotope records (Kaufman and Knoll, 1995; Halverson and Hurtgen, 2007) and indications of a transition from an anoxic to a partially oxygenated deeper ocean in the aftermath of the Marinoan glaciation (Planavsky et al., 2010). These changes can arguably be best studied in marine sediments from the Yangtze Platform, South China, that cover the critical time span from the Cryogenian to the Precambrian-Cambrian (PCC) boundary which saw the appearance of the first macroscopic fossils (e.g., Yin et al., 2007) For this reason, it may also be an ideal place to evaluate whether $\mathrm{Cd}$ isotopes in marine sediments can be used as a palaeo-productivity proxy. To test this, we analysed shallow-water carbonates from the Ediacaran Xiaofenghe section that was deposited on the S-SE-facing passive margin of the Yangtze Craton and hosts abundant fossil assemblages of multicellular life from the Doushantuo and Dengying Formations (Xiao et al., 2012).

Stable isotope fractionation of $\mathrm{Cd}$ is a new proxy for studying biogeochemical cycling of micronutrients in the present-day oceans (Lacan et al., 2006; Ripperger et al., 2007; Abouchami et al., 2011) and in sedimentary archives, such as Fe-Mn crusts (Schmitt, et al., 2009; Horner et al., 2010). Seawater profiles of $\mathrm{Cd}$ show a nutrient-like behaviour, with strong near-surface depletions and deep-water enrichments, mimicking profiles of macronutrient phosphate (Boyle et al., 1976). The surface depletions in Cd are associated with "heavier" Cd stable isotopic compositions, which are thought to be due to preferential uptake of the "lighter" isotopes during incorporation of Cd by phytoplankton (Lacan et al., 2006; Ripperger et al., 2007; Abouchami et al., 2011). At the moment, there is no general understanding of why cadmium, which is toxic to most life, should be taken up. Zinc is required by all organisms and in the case of phytoplankton is the cofactor in zinc carbonic anhydrase ( $\mathrm{Zn}-\mathrm{CA})$, an enzyme involved in photosynthesis. Under zinc-poor conditions, Cd can substitute for $\mathrm{Zn}$ in $\mathrm{Zn}-\mathrm{CA}$ and, furthermore, Price and Morel (1990) have described a cadmium-bearing carbonic anhydrase variant (Cd-CA) with the same functionality; but $\mathrm{Cd}-\mathrm{CA}$ is only known from a few diatom species (Park et al., 2007) whose first appearance lies in the Mesozoic. Studying Cd systematics in Proterozoic sediments could therefore possibly provide insights into the lineage of the CA enzymes. It is equally possible that cadmium fulfills no biological role at all, in most cases, and that $\mathrm{Cd}$ uptake is driven by absorption onto organic matter or that $\mathrm{Cd}$ is pumped into the cell through the membrane, mistaken for $\mathrm{Zn}$.

Similarly, it remains unclear what the precise mechanism is for causing the isotopic fractionation of Cd during uptake from seawater. Pure inorganic absorption (Wasylenki et al., 2014), partitioning into calcite (Horner et al., 2011) and biological utilisation are known to favour the light isotopes, which would all be consistent with the observation of heavy $\mathrm{Cd}$ in surface seawaters. But irrespective of these uncertainties concerning the reason for uptake and site of fractionation, 
Cd isotopes can be used as a convincing proxy for productivity in surface waters in the sense that the greater the depletion in Cd (and macronutrients like phosphate), the isotopically heavier the $\mathrm{Cd}$ becomes that is left behind in seawater.

So far the oldest sediments analysed for their $\mathrm{Cd}$ isotopic compositions are Permian in age (Georgiev et al., 2015). Studying Cd isotopes in our Ediacaran-age carbonates could potentially document palaeo-seawater biogeochemical cycling of cadmium, providing insights into evolution of multicellular life at the PCC boundary. Furthermore, Cd isotopes may also be used to support interpretations from other bio-available isotopic tracers. In recent years, $\delta^{15} \mathrm{~N}$ has provided important insights into palaeo-ocean nutrient-cycling and redox conditions (Ader et al., 2014). As the $\delta^{15} \mathrm{~N}$ proxy is often affected by post-depositional modification (Bebout and Fogel, 1992), combining with Cd isotopes is potentially usefu in screening such datasets. Nevertheless, when analysing Cd isotopes in ancient sediments, other factors that may mask the true seawater signal must be carefully evaluated, such as fluid flow alteration and additional inorganic fractionation processes (e.g., variable salinity, authigenic sulphide precipitation).

\section{Results and Discussion}

Stable isotope measurements of $\mathrm{C}$ and $\mathrm{N}$ were performed on carbonates and bulk rock using standard methods. Stable $\mathrm{Cd}$ isotope compositions on acetic acid leachates from alteration-free carbonates were determined using a double-spike method and TIMS (see Schmitt et al., 2009; Abouchami et al., 2011; detailed analytical protocols in Supplementary Information).

The $\delta^{13} \mathrm{C}_{\text {org }}$ values obtained up the stratigraphic section remain relatively constant at $-28 \%$ before decreasing to $-34 \%$ in black shales of the middle Doushantuo, where TOC values increase to $1.1 \%$. These values are characteristic of enhanced planktonic productivity and remineralisation and resemble previously published data from the Doushantuo (e.g., Ishikawa et al., 2013). In the cap dolostones $\delta^{13} \mathrm{C}_{\text {org }}$ and $\delta^{13} \mathrm{C}_{\text {carb }}$ are decoupled $\left(\Delta^{13} \mathrm{C}=25\right.$ vs. 33 in overlying strata, Fig. 1c) and this is mimicked in a shift in average bulk $\delta^{15} \mathrm{~N}$ values from $+1.6 \%$ o to $+4.2 \%$ o (Fig. 1f), possibly indicating a change in the nutrient regime to a $\mathrm{NO}_{3}{ }^{-}$-dominated marine environment (Ader et al., 2014). In the Doushantuo Formation, nitrate presumably became the main utilised nutrient for primary producers while $\mathrm{N}_{2}$ partly remained unused in surface-waters, where incomplete denitrification shifts seawater to higher $\delta^{15} \mathrm{~N}$. This may possibly be attributable to increased oxygen availability, which is essential for organic matter (OM) remineralisation via nitrification-denitrification (Canfield et al., 2010); however, smaller second-order variations in the $\delta^{15} \mathrm{~N}$ curve might simply be due to alteration.

Cadmium isotopic compositions in carbonates vary by more than four $\varepsilon^{112 / 110} \mathrm{Cd}$ units and show no definite correlation with $\mathrm{Cd}$ (Fig. 2a) nor K concentrations (see Fig. S-1b). Cadmium concentrations in detrital material are very

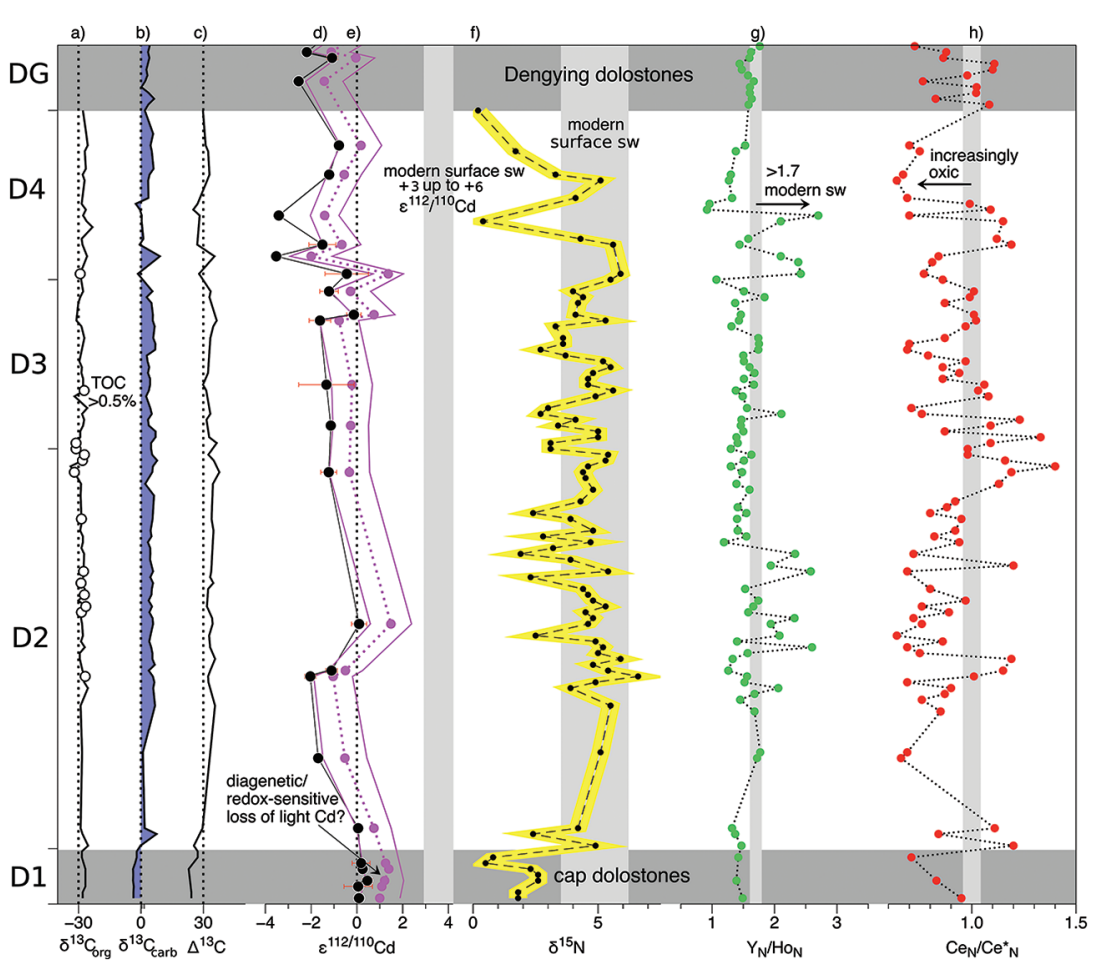

Figure 1 Isotope and concentration data from Xiaofenghe section. Samples are drawn in equidistance according to their sample numbers in Table 1 for better visibility. (a-c) Carbon isotope data. (d) Carbonate $\varepsilon^{112 / 110} \mathrm{Cd}$ (error bars $=2 \sigma$ ), grey bar $=$ discrimination line for modern seawater values (Ripperger et al., 2007). (e) Salinity-corrected $\varepsilon^{122 / 110} \mathrm{Cd}$ of seawater. (f) Bulk $\delta^{15} \mathrm{~N}$, yellow envelope $=0.2 \%$ uncertainty, grey bar $=$ modern surface water. $(\mathbf{g}, \mathbf{h})$ Shale-normalised $\mathrm{Y} / \mathrm{Ho}$ and $\mathrm{Ce} / \mathrm{Ce}$ in carbonates, grey bars represents discrimination of modern seawater values (Bau et al., 1995) and negative/positive Ce anomalies.

low, so the effect of detrital input on $\varepsilon^{112 / 110} \mathrm{Cd}$ is likely negligible in extent. Late fluid-flow overprint is typical for many Neoproterozoic carbonates and may also have altered the Cd isotope signatures. However, Hohl et al. (2015) have shown that, with the exception of the cap dolostones, carbonates at Xiaofenghe were not much affected by fluids (SEM pre-screening, no co-variation of $\delta^{18} \mathrm{O}$ with ${ }^{87} \mathrm{Sr} /{ }^{86} \mathrm{Sr}$ or $\mathrm{Mn} / \mathrm{Sr}$ ); thus, their trace element budgets and isotopic compositions most likely reflect partitioning and incorporation from Ediacaran seawater. In the cap dolostones $\varepsilon^{112 / 110} \mathrm{Cd}>0$, while low $\mathrm{Cd}$ and $\mathrm{N}$ concentrations (Fig. S-1d) correlate with light $\delta^{13} \mathrm{C}_{\text {carb }}$ (Fig. 1c) and high Mn/Sr ratios (see Fig. S-1a), commonly used as indicators of fluid overprinting (Brand and Veizer, 1980). 
Table 1 Stable isotope data; $\mathrm{N}$ and $\mathrm{C}$ concentrations; assorted shale normalised REE ratios and $\mathrm{Mn}$ enrichments relative to Cal-s from Hohl et al. (2015b). D1-D4 = Doushantuo Fm. DG = Dengying Fm.

\begin{tabular}{|c|c|c|c|c|c|c|c|c|c|}
\hline sample & $\begin{array}{l}\text { Height } \\
\text { [m] }\end{array}$ & Member & $\delta^{13} C_{\text {carb }}{ }^{1}$ & $\delta^{18} \mathrm{O}_{\text {carb }}{ }^{1}$ & $\delta^{13}$ Corg & $\Delta \delta^{13}$ & $\begin{array}{c}\varepsilon^{112 / 110} \\
\mathrm{Cd}^{2}\end{array}$ & 2SE & $\begin{array}{c}\mathrm{Cd} \\
{[\mu \mathrm{g} / \mathrm{g}]}\end{array}$ \\
\hline 1 & 0.2 & D1 & -3.8 & -6.7 & -28 & 24.2 & 0.09 & 0.2 & 0.06 \\
\hline 2 & 0.75 & D1 & & & -26.7 & & 0.06 & 0.62 & 0.1 \\
\hline 3 & 1.6 & D1 & -3.7 & -7.7 & & & 0.46 & 0.23 & 0.03 \\
\hline 4 & 2.3 & D1 & -3.8 & -7.3 & -26.7 & 22.9 & 0.25 & 0.19 & 0.07 \\
\hline 5 & 3.85 & D1 & -3.2 & -6.9 & & & 0.18 & 0.39 & 0.03 \\
\hline 6 & 4.8 & D1 & -1.2 & -12.3 & -28.4 & 27.2 & & & \\
\hline 7 & 6.65 & D2 & 0.0 & -6.8 & -25.4 & 25.4 & & & \\
\hline 8 & 12.1 & D2 & & & -28.1 & & & & \\
\hline 9 & 22 & D2 & 0.6 & -10.4 & -29 & 29.6 & 1.07 & 0.35 & 0.03 \\
\hline 10 & 36 & D2 & & & & & -1.69 & 0.09 & 0.2 \\
\hline 11 & 37.5 & D2 & & & -28.3 & & & & \\
\hline 12 & 65 & D2 & 6.5 & -6.1 & -29 & 35.5 & & & \\
\hline 13 & 73.7 & D2 & & & -25.5 & & & & \\
\hline 14 & 75 & D2 & 5.1 & -4.8 & -27.3 & 32.4 & & & \\
\hline 15 & 75.6 & D2 & 5.4 & -4.4 & -26.8 & 32.2 & -2.04 & 0.25 & 0.04 \\
\hline 16 & 76 & D2 & 5.2 & -4.5 & -28.1 & 33.3 & -1.11 & 0.25 & 0.05 \\
\hline 17 & 76.5 & D2 & & & & & & & \\
\hline 18 & 77.5 & D2 & 6.2 & -4.1 & -29.4 & 35.6 & & & \\
\hline 19 & 77.5 & D2 & & & -29.4 & & & & \\
\hline 20 & 79.5 & D2 & 6.2 & -4.1 & -27 & 33.2 & & & \\
\hline 21 & 80.5 & D2 & & & -28.8 & & & & \\
\hline 22 & 81.5 & D2 & 4.8 & -5.0 & -27.9 & 32.7 & & & \\
\hline 23 & 84.5 & D2 & 6.1 & -1.6 & -28.3 & 34.4 & 0.1 & 0.33 & 0.02 \\
\hline 24 & 85 & D2 & 5.4 & -4.4 & -28.8 & 34.2 & & & \\
\hline 25 & 86 & D2 & & & -28.6 & & & & \\
\hline 26 & 87.2 & D2 & 5.2 & -3.9 & -26.5 & 31.7 & & & \\
\hline 27 & 88 & D2 & & & -30.1 & & & & \\
\hline 28 & 88.7 & D2 & & & -27.2 & & & & \\
\hline 29 & 89 & D2 & 5.2 & -4.0 & -27 & 32.2 & & & \\
\hline 30 & 91.2 & D2 & 5.5 & -3.3 & -27.7 & 33.2 & & & \\
\hline 31 & 91.7 & D2 & 6.1 & -2.9 & -27.6 & 33.7 & & & \\
\hline 32 & 92.5 & D2 & & & & & & & \\
\hline 33 & 93 & D2 & & & -27.3 & & & & \\
\hline 34 & 96.2 & D2 & & & -28.6 & & & & \\
\hline 35 & 98 & D2 & & & -27.9 & & & & \\
\hline 36 & 99 & D2 & & & -27.9 & & & & \\
\hline 37 & 99.85 & D2 & 5.6 & -2.6 & -29.2 & 34.8 & & & \\
\hline 38 & 101.5 & D2 & 5.3 & -3.4 & -28.6 & 33.9 & & & \\
\hline 39 & 101.85 & D2 & 5.8 & -2.4 & & & & & \\
\hline 40 & 102 & D2 & 6.3 & -1.3 & & & & & \\
\hline 41 & 102.4 & D2 & & & & & & & \\
\hline 42 & 102.6 & D2 & & & & & & & \\
\hline 43 & 102.7 & D2 & 5.6 & -1.5 & -32 & 37.6 & -1.23 & 0.35 & 0.1 \\
\hline
\end{tabular}

$1 \mathrm{O}$ and $\mathrm{C}$ isotopic data relative to VPDB. 2 Cd relative to NIST SRM 3108. ${ }^{3} \mathrm{~N}$ relative to Air:

\begin{tabular}{|c|c|c|c|c|c|c|c|c|}
\hline$\delta^{15} \mathbf{N}^{3}$ & $\mathrm{~N}$ [wt. \%] & $\begin{array}{c}\text { TOC } \\
\text { [wt.\%] }\end{array}$ & $\mathrm{C} / \mathrm{N}$ & $\begin{array}{l}\sum \mathrm{REE} \\
{[\mu \mathrm{g} / \mathrm{g}]}\end{array}$ & $\mathrm{Ce} / \mathrm{Ce}^{* 4}$ & $\mathrm{Y}_{\mathrm{N}} / \mathrm{Ho}_{\mathrm{N}}$ & $\operatorname{Pr}_{N} / Y b_{N}$ & $\begin{array}{c}\mathrm{EF} \\
\mathrm{Mn}_{(\mathrm{Cal}-S)}\end{array}$ \\
\hline 1.8 & 0.003 & $\begin{array}{l}0.01 \\
0.11\end{array}$ & 2.7 & 16 & 0.95 & 1.5 & 0.97 & 48 \\
\hline 2.6 & 0.006 & & & 27 & 0.83 & 1.4 & 1.1 & 30 \\
\hline 2.3 & 0.005 & 0.0001 & 0.02 & & & & & \\
\hline 0.5 & 0.011 & & & & & & & \\
\hline 0.8 & 0.004 & 0.06 & 15 & 2.1 & 0.71 & 1.4 & 0.97 & 1 \\
\hline 4.9 & 0.066 & 0.02 & 0.2 & 13 & 1.2 & 1.5 & 1.05 & 496 \\
\hline 2.4 & 0.008 & 0.25 & 32 & 4.9 & 0.84 & 1.4 & 0.85 & 7.8 \\
\hline \multirow[t]{2}{*}{4.2} & 0.011 & 0.12 & 11 & 2.5 & 1.11 & 1.3 & 0.41 & 8.9 \\
\hline & & & & 2.2 & 0.66 & 1.7 & 0.57 & 2.1 \\
\hline 5.1 & 0.014 & 0.21 & 15 & 1.7 & 0.69 & 1.8 & 0.49 & 2.9 \\
\hline 5.5 & 0.028 & 0.12 & 4.4 & 0.33 & & & & 0.71 \\
\hline 3.9 & 0.017 & & & 3.8 & 0.9 & 2.1 & 0.77 & 6.4 \\
\hline 4.9 & 0.092 & & & 0.33 & 0.69 & 1.5 & 0.4 & 0.14 \\
\hline 6.6 & 0.13 & 0.61 & 4.7 & 2.9 & 1.01 & 1.6 & 0.58 & 1.7 \\
\hline 5.4 & 0.034 & 0.22 & 6.6 & 2.9 & 1.15 & 1.3 & 0.62 & 5.2 \\
\hline 4.8 & 0.023 & & & & & & & \\
\hline 5.9 & 0.046 & 0.43 & 9.3 & 3.4 & 1.19 & 1.3 & 0.63 & 5.2 \\
\hline 5.0 & 0.032 & 0.42 & 13 & 1.4 & 0.75 & 1.6 & 0.49 & 4.8 \\
\hline 5.2 & 0.044 & 0.41 & 9.4 & 1.7 & 0.69 & 2.6 & 0.37 & 1.7 \\
\hline 4.9 & 0.049 & 0.38 & 7.7 & 13 & 0.86 & 1.4 & 1.31 & 3.8 \\
\hline 2.5 & 0.092 & 0.01 & 0.1 & 3.2 & 0.64 & 2.1 & 0.5 & 1.6 \\
\hline 4.6 & 0.031 & 0.45 & 14 & 3.6 & 0.76 & 1.9 & 0.65 & 2.2 \\
\hline 4.8 & 0.034 & 0.48 & 14 & 1.9 & 0.72 & 2.3 & 0.36 & 2.5 \\
\hline 4.5 & 0.067 & 0.92 & 14 & 6.4 & 0.89 & 1.6 & 0.81 & 2.1 \\
\hline 5.3 & 0.044 & 0.71 & 16 & 6.9 & 0.76 & 1.7 & 0.89 & 2.5 \\
\hline 4.8 & 0.037 & 0.36 & 10 & 7.3 & 0.97 & 1.7 & 0.7 & 2.2 \\
\hline 4.6 & 0.048 & 0.71 & 15 & & & & & \\
\hline 4.4 & 0.044 & & & 6.7 & 0.8 & 1.5 & 0.91 & 1.1 \\
\hline 2.3 & 0.017 & 0.29 & 17 & & & & & \\
\hline 5.4 & 0.087 & 0.77 & 8.9 & 1.3 & 0.69 & 2.58 & 0.49 & 2.3 \\
\hline 3.9 & 0.020 & & & & & & & \\
\hline 1.9 & 0.011 & 0.26 & 23 & 2.4 & 0.72 & 2.3 & 0.57 & 1.4 \\
\hline 3.2 & 0.014 & 0.35 & 25 & & & & & \\
\hline 4.7 & 0.04 & 0.35 & 8.7 & 29 & 0.94 & 1.2 & 0.92 & 3.2 \\
\hline 2.8 & 0.011 & 0.15 & 13 & 16 & 0.82 & 1.6 & 1.52 & 3.1 \\
\hline 4.8 & 0.06 & 0.32 & 5.3 & 10 & 0.92 & 1.4 & 1.36 & 2.9 \\
\hline 3.9 & 0.019 & 0.65 & 34 & 3.5 & 0.95 & 1.4 & 0.82 & 3.6 \\
\hline 2.4 & 0.017 & 0.28 & 16 & 6 & 0.8 & 1.6 & 1.22 & 4 \\
\hline 4.3 & 0.045 & & & 9.4 & 0.92 & & & 2.5 \\
\hline 4.8 & 0.094 & 0.95 & 10 & & & 1.6 & 0.8 & \\
\hline 4.5 & 0.097 & 0.4 & 4.1 & & & & 0.63 & \\
\hline 4.4 & 0.085 & 1 & 12 & 5.8 & 1.19 & 1.5 & 0.73 & 4.0 \\
\hline
\end{tabular}




\begin{tabular}{|c|c|c|c|c|c|c|c|c|c|}
\hline sample & $\begin{array}{l}\text { Height } \\
\text { [m] }\end{array}$ & Member & $\delta^{13} \mathrm{C}_{\mathrm{carb}}{ }^{1}$ & $\delta^{18} \mathbf{O}_{\text {carb }}{ }^{1}$ & $\delta^{13}$ Corg & $\Delta \delta^{13}$ & $\begin{array}{c}\varepsilon^{112 / 110} \\
C^{2}{ }^{2}\end{array}$ & 2SE & $\begin{array}{c}\mathrm{Cd} \\
{[\mu \mathrm{g} / \mathrm{g}]}\end{array}$ \\
\hline 44 & 103 & D2 & & & -33.8 & & & & \\
\hline 45 & 103 & D2 & 6.8 & -1.1 & -28.1 & 34.9 & & & \\
\hline 46 & 103 & D2 & 5.1 & -2.1 & -27.2 & 32.3 & & & \\
\hline 47 & 103.5 & D3 & & & -31.2 & & & & \\
\hline 48 & 103.5 & D3 & 5.2 & -3.4 & -31.2 & 36.4 & & & \\
\hline 49 & 104 & D3 & 3.5 & -2.8 & -29.2 & 32.7 & & & \\
\hline 50 & 104 & D3 & & & -29.2 & & & & \\
\hline 51 & 104.25 & D3 & & & -27.8 & & -1.14 & 0.12 & 0.4 \\
\hline 52 & 104.5 & D3 & 4.2 & -2.2 & -27.3 & 31.5 & & & \\
\hline 53 & 104.5 & D3 & 5.0 & -4.9 & -28.3 & 33.3 & & & \\
\hline 54 & 105.5 & D3 & & & -25.8 & & & & \\
\hline 55 & 108.15 & D3 & & & -31.9 & & & & \\
\hline 56 & 108.7 & D3 & 4.0 & -1.0 & -27.3 & 31.3 & & & \\
\hline 57 & 109.5 & D3 & 2.0 & -6.3 & -27.8 & 29.8 & -1.33 & 1.21 & 0.03 \\
\hline 58 & 109.5 & D3 & & & -27.8 & & & & \\
\hline 59 & 110 & D3 & 3.6 & -6.8 & -28.6 & 32.2 & & & \\
\hline 60 & 110.5 & D3 & & & -28.4 & & & & \\
\hline 61 & 111.1 & D3 & 2.9 & -6.8 & -29.6 & 32.5 & & & \\
\hline 62 & 112.2 & D3 & 2.8 & -8.6 & & & & & \\
\hline 63 & 113.9 & D3 & 6.6 & -5.4 & & & & & \\
\hline 64 & 116 & D3 & 6.9 & -4.6 & & & & & \\
\hline 65 & 118.5 & D3 & 6.1 & -6.4 & -27.2 & 33.3 & & & \\
\hline 66 & 123.8 & D3 & 6.1 & -2.3 & -28.6 & 34.7 & & & \\
\hline 67 & 125 & D3 & 5.4 & -3.2 & -31 & 36.4 & -1.62 & 0.47 & 0.02 \\
\hline 68 & 130 & D3 & 5.7 & -4.9 & & & -0.13 & 0.31 & 0.08 \\
\hline 69 & 133.5 & D3 & & & -30.1 & & & & \\
\hline 70 & 135 & D3 & 3.7 & -7.5 & -29.2 & 32.9 & & & \\
\hline 71 & 136 & D3 & 5.6 & -6.7 & -27.8 & 33.4 & -1.22 & 0.41 & 0.01 \\
\hline 72 & 142 & D4 & & & & & & & \\
\hline 73 & 142.4 & D4 & -1.3 & -0.9 & -29.2 & 27.9 & -0.44 & 0.95 & 0.04 \\
\hline 74 & 156.5 & D4 & -1.1 & -0.4 & -28.1 & 27.0 & -1.51 & 0.59 & 0.01 \\
\hline 75 & 158 & D4 & & & -28.5 & & & & \\
\hline 76 & 166 & D4 & & & -26.1 & & & & \\
\hline 77 & 167 & D4 & 0.4 & & -27.8 & 28.2 & -3.41 & 0.1 & 0.18 \\
\hline 78 & 172.2 & D4 & 3.3 & -1.8 & & & & & \\
\hline 79 & 174 & D4 & 4.7 & -3.0 & -27.1 & 31.8 & & & \\
\hline 80 & 175.5 & D4 & 4.9 & -1.7 & -28 & 32.9 & -1.21 & 0.17 & 0.11 \\
\hline 81 & 179.8 & D4 & 5.2 & -4.5 & -27 & 32.2 & & & \\
\hline 82 & 180 & D4 & 5.4 & -5.3 & -25.6 & 31.0 & -0.79 & 0.12 & 0.07 \\
\hline 83 & 194 & D4 & 1.8 & & -27.9 & 29.7 & & & \\
\hline 84 & 203 & DG & 0.9 & -2.8 & & & -2.55 & 0.14 & 0.13 \\
\hline 85 & 209 & DG & & & & & -1.08 & 0.24 & 0.08 \\
\hline 86 & 213 & DG & 3.3 & -4.0 & & & -2.19 & 0.10 & 0.07 \\
\hline 87 & 217 & DG & 3.2 & -3.4 & & & -1.55 & 0.17 & 0.07 \\
\hline
\end{tabular}

\begin{tabular}{|c|c|c|c|c|c|c|c|c|}
\hline$\delta^{15} \mathbf{N}^{3}$ & N [wt. \%] & $\begin{array}{c}\text { TOC } \\
\text { [wt.\%] }\end{array}$ & $\mathrm{C} / \mathrm{N}$ & $\begin{array}{l}\sum \text { REE } \\
{[\mu \mathrm{g} / \mathrm{g}]}\end{array}$ & $\mathrm{Ce} / \mathrm{Ce}^{* 4}$ & $\mathrm{Y}_{\mathrm{N}} / \mathrm{Ho}_{\mathrm{N}}$ & $\mathrm{Pr}_{\mathrm{N}} / \mathrm{Yb}_{\mathrm{N}}$ & $\begin{array}{c}\text { EF } \\
\mathrm{Mn}_{\text {(Cal-S) }}\end{array}$ \\
\hline 4.6 & 0.051 & 0.21 & 4.1 & 8.8 & 1.4 & 1.3 & 0.83 & 2.9 \\
\hline 5.3 & 0.137 & 0.97 & 7.1 & 44 & 1.16 & 1.5 & 0.75 & 2.1 \\
\hline 5.4 & 0.126 & 0.89 & 7.1 & 11 & 0.98 & 1.6 & 0.7 & 2.4 \\
\hline 3.1 & 0.015 & 0.59 & 39 & 3.9 & 0.98 & 1.3 & 1.07 & 1.2 \\
\hline 3.1 & 0.015 & 0.59 & 39 & 3.5 & 1.09 & 1.4 & 0.84 & 3.5 \\
\hline 5.0 & 0.029 & 0.11 & 3.9 & 14 & 1.33 & 1.4 & 1.32 & \\
\hline 5.0 & 0.029 & 0.11 & 3.9 & 2.6 & 0.87 & 1.5 & 0.94 & 1.6 \\
\hline 3.4 & 0.015 & 0.17 & 11 & 2.7 & 1.09 & 1.5 & 1 & 3.4 \\
\hline 4.1 & 0.012 & 0.15 & 12 & 3.4 & 1.23 & 1.5 & 0.73 & 5.3 \\
\hline 2.7 & 0.018 & 0.23 & 13 & 2.9 & 0.76 & 2.1 & 0.31 & 2.3 \\
\hline 3.0 & 0.009 & 0.43 & 48 & 2.9 & 0.71 & 1.6 & 0.75 & 1.5 \\
\hline 4.9 & 0.039 & 0.21 & 5.5 & 5.7 & 1.08 & 1.5 & 1.2 & 9.5 \\
\hline 5.6 & 0.126 & 0.56 & 4.4 & 15 & 1.03 & 1.4 & 0.97 & 2.4 \\
\hline 4.6 & 0.013 & 0.13 & 10 & 8.6 & 1.06 & 1.7 & 1.39 & 34 \\
\hline 4.6 & 0.013 & & & 11 & 0.86 & 1.5 & 1.29 & 4.4 \\
\hline 4.8 & 0.01 & 0.1 & 9.6 & 14 & 0.94 & 1.7 & 2.59 & 116 \\
\hline 5.5 & 0.01 & 0.09 & 8.7 & 16 & 0.86 & 1.6 & 3.24 & 257 \\
\hline 5.2 & 0.015 & 0.14 & 9.3 & 11 & 0.97 & 1.5 & 2.23 & 31 \\
\hline 3.7 & 0.008 & & & 8.3 & 0.79 & 1.5 & 2.03 & 74 \\
\hline 2.7 & 0.007 & 0.05 & 7.3 & 3.5 & 0.69 & 1.7 & 1.83 & \\
\hline 3.6 & 0.010 & & & 3.0 & 0.7 & 1.8 & 1.23 & \\
\hline 3.6 & 0.009 & 0.07 & 8.0 & 6.7 & 0.87 & 1.7 & 1.37 & 162 \\
\hline 3.3 & 0.012 & 0.17 & 14.5 & 11 & 0.97 & 1.3 & 1.67 & 7 \\
\hline 5.3 & 0.018 & 0.24 & 13.1 & 10 & 1.02 & 1.4 & 1.98 & 19 \\
\hline 4.1 & 0.009 & & & 12 & 1.01 & 1.5 & 2.97 & 60 \\
\hline 4.2 & 0.008 & 0.16 & 20 & 9.0 & 0.87 & 1.4 & 2.21 & 32 \\
\hline 4.4 & 0.007 & 0.31 & 44 & 16 & 0.99 & 1.8 & 1.17 & 2 \\
\hline 4.0 & 0.007 & 0.13 & 18 & 11 & 1.01 & 1.5 & 2.3 & 10 \\
\hline 5.5 & 0.017 & & & 12 & 0.86 & 1.1 & 1.04 & 2.1 \\
\hline 5.9 & 0.048 & 1.61 & 34 & 1.9 & 0.77 & 2.4 & 0.38 & 0.1 \\
\hline 5.6 & 0.023 & 0.13 & 5.5 & 7.1 & 1.2 & 1.4 & 0.42 & 1.6 \\
\hline 4.3 & 0.008 & 0.07 & 8.1 & 7.4 & 1.1 & 1.6 & 0.54 & 3.5 \\
\hline \multirow{2}{*}{0.4} & 0.008 & 0.04 & 5.5 & 1.9 & 1.2 & 2.1 & 0.19 & 0.6 \\
\hline & & 0.02 & & 0.99 & 0.7 & 2.7 & 0.23 & 0.2 \\
\hline 4.1 & 0.01 & 0.18 & 18 & 9.1 & 0.69 & 1.3 & 0.87 & 2.7 \\
\hline 5.1 & 0.022 & 0.09 & 4 & 14.5 & 0.64 & 1.3 & 1.4 & 4.3 \\
\hline 3.3 & 0.008 & 0.03 & 3.3 & 11 & 0.67 & 1.3 & 1.7 & 2.6 \\
\hline \multirow[t]{2}{*}{1.7} & 0.005 & 0.14 & 29 & 13 & 0.75 & 1.4 & 2 & 1.9 \\
\hline & & 0.09 & & 13 & 0.7 & 1.5 & 2 & 3.5 \\
\hline \multirow[t]{5}{*}{0.2} & 0.008 & 0.09 & 12 & & & & & \\
\hline & & & & 0.43 & 0.76 & 1.7 & 0.15 & 1.9 \\
\hline & & & & 0.56 & 0.86 & 1.6 & 0.2 & 5.7 \\
\hline & & & & 0.51 & 0.88 & 1.6 & 0.18 & 2.7 \\
\hline & & & & 0.69 & 0.83 & 1.8 & 0.23 & 2.5 \\
\hline
\end{tabular}



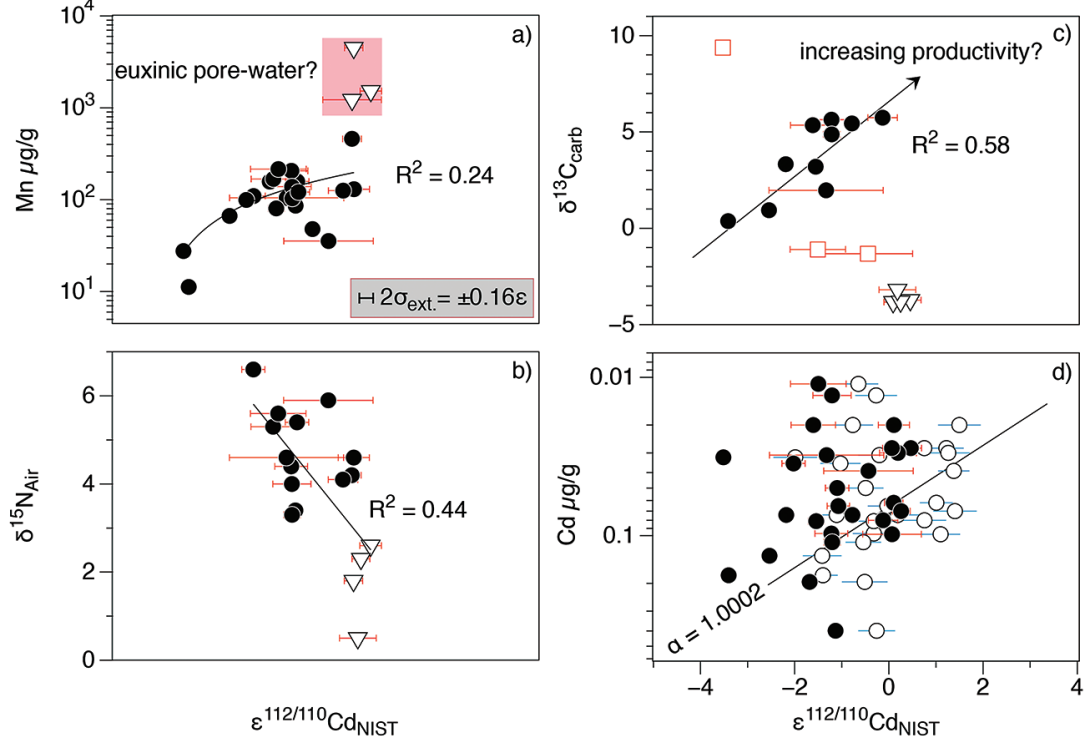

Figure 2 (a) Mn vs. $\varepsilon^{122 / 110} \mathrm{Cd}$. Cap dolomites (open triangles) have high Mn concentrations and exhibit positive $\varepsilon^{112 / 110} \mathrm{Cd}$. (b) $\mathrm{N}$ vs. Cd isotopic compositions reveal negative trend. (c) Carbon vs. Cd isotopic compositions show slight positive correlation when extreme values (red squares) and cap dolomites are excluded. (d) Cd concentrations vs. . $^{12 / 1110} \mathrm{Cd}$ values scatter along modern Southern ocean fractionation line (Abouchami et al., 2011), open circles represent coeval calculated $\varepsilon^{112 / 110} \mathrm{Cd}_{\mathrm{sw}}$; note that the seawater $\mathrm{Cd}$ concentrations cannot be estimated from those measured in the carbonates.

As has been discussed above, there is still no consensus as to why cadmium is taken up by phytoplankton in the surface ocean, nor at which step (or steps) in this process the isotope fractionation takes place. Nevertheless, it is clear that $\varepsilon^{112 / 110} \mathrm{Cd}$ is a good indicator of biological productivity in the modern surface ocean: $\mathrm{Cd}$ uptake into OM leads to a depletion of light $\mathrm{Cd}$ in the photic zone. But can Cd isotopes in a marine sedimentary archive be used as a palaeo-productivity proxy? Under closed-system conditions (e.g., a restricted basin) Ediacaran carbonates precipitating in biologically productive environments would be expected to have higher (heavier) $\varepsilon^{112 / 110} \mathrm{Cd}$ and $\delta^{13} \mathrm{C}_{\mathrm{c} a r b}$. The Xiaofenghe carbonates do exhibit a positive correlation between $\varepsilon^{112 / 110} \mathrm{Cd}$ and $\delta^{13} \mathrm{C}_{\text {carb }}$ (Fig. $2 \mathrm{c}$ ), consistent with increasing bio-productivity. However, this correlation only exists once light $\delta^{13} \mathrm{C}_{\text {carb }}$ values from the basal Doushantuo (oxidation of a ${ }^{12} \mathrm{C}$-enriched hydrocarbon source) and one sample with extremely heavy $\delta^{13} \mathrm{C}_{\text {carb }}$ of +9 (presumably due to evaporation) are excluded.

The $\varepsilon^{112 / 110} \mathrm{Cd}$ and $\delta^{15} \mathrm{~N}$ curves in Figure 1 show an anti-correlation in some parts of the profile - for example, higher $\varepsilon^{112 / 110} \mathrm{Cd}$ in the cap dolomites with $\delta^{15} \mathrm{~N}$ having low but positive values (Fig. $2 b$ ). This trend would be consistent with strong $\mathrm{N}_{2}$ fixation by diazotrophs in the euphotic zone (Sachs and Repeta, 1999). Cyanobacteria blooms of this sort are often documented from redox-stratified basins (Struck et al., 2004), and recent work by Georgiev et al. (2015) has highlighted the redox control on $\mathrm{Cd}$ distribution in late Permian marine sediments. $\mathrm{Cd}$ is a not a redox-sensitive element per se, but $\mathrm{Cd}$ bound to $\mathrm{OM}$ will become enriched in anoxic sediments whenever oxidation of OM is inhibited.

Cadmium may also be co-precipitated with sulphides under euxinic conditions (Framson and Leckie, 1978), such as prevail in the deeper parts of the Black Sea today, where $\mathrm{Cd}$ is removed almost quantitatively from the water column and enriched in bottom sediments (Lewis and Landing, 1992). The effect of authigenic sulphides on Cd isotope fractionation is still not well understood. Cadmium isotope data on hydrothermal sulphide ores (Schmitt et al., 2009; Wen et al., 2016) and quantum chemical calculations (Yang et al., 2015) suggest that the light isotopes of $\mathrm{Cd}$ would be preferentially bound into the sulphide phase. On the other hand, Rolison et al. (2015) did not find the high $\varepsilon^{112 / 110} \mathrm{Cd}$ in the deeper parts of the Black Sea water column that one would expect. All in all this means that sulphide-bearing anoxic sediments should most likely have higher $\mathrm{Cd}$ concentrations and more negative $\varepsilon^{112 / 110} \mathrm{Cd}$ than those of oxic surface waters.

In the Xiaofenghe carbonates, Mn enrichments correlate with high $\varepsilon^{112 / 110} \mathrm{Cd}$ (Fig. 2a). As $\mathrm{Mn}$ in oxic waters is usually incorporated into Mn oxyhydroxides, such enrichments are likely to reflect reduction of $\mathrm{Mn}$ to the 2+ state at the anoxic pore-water sediment interface and subsequent incorporation into $\mathrm{CaCO}_{3}$ (Thomson et al, 1986). The cap dolomites exhibit Mn enrichments, more positive $\varepsilon^{112 / 110} \mathrm{Cd}$ and low $\mathrm{Cd}$ concentrations. Furthermore, sedimentary sulphides ranging from 10 to $40 \mu \mathrm{m}$ in size are abundant in thin section (see Fig. $\mathrm{S}-2)$. The fact that $\mathrm{Ce}$ anomalies are negative $\left(\mathrm{Ce} / \mathrm{Ce}^{*}<0.9\right)$ in shallow Yangtze Platform cap dolomites (Fig. 1h) suggests that early Ediacaran surface-waters were already oxic; nonetheless, pore-waters may still have been anoxic, leading to the formation of sulphides and Mn-rich carbonates. In summary, incorporation of light $\mathrm{Cd}$ into sulphides may raise $\varepsilon^{112 / 110} \mathrm{Cd}$ in associated carbonates, and might have affected the Xiaofenghe cap dolomites.

Experiments on precipitation of inorganic calcite from seawater by Horner et al. (2011) show that the seawater/calcite fractionation factor for $\mathrm{Cd}$ is insensitive to temperature, $\mathrm{Mg}$ concentration and precipitation rate. By contrast, precipitation of calcites from freshwater does not lead to any Cd isotopic fractionation, presumably as a result of ion blocking on mineral surface sites. Since these experiments by Horner et al. (2011) used only seawater and freshwater end-members, we will assume to first order that $\mathrm{Cd}$ isotope fractionation into calcite depends linearly on salinity. Using $\mathrm{Y} / \mathrm{H}$ o ratios as an indicator of palaeo-salinity we are then able to calculate $\varepsilon^{112 / 110} \mathrm{Cd}$ for seawater in equilibrium with the Xiaofenghe carbonates (see Fig. S-3 for more details). The resulting $\varepsilon^{112 / 110} \mathrm{Cd}_{\mathrm{sw}}$ range between -2 and +1.5 (Fig. 1e), which is still slightly lower than the lower bound of modern surface seawater $\varepsilon^{112 / 110} \mathrm{Cd}$ but scatter around the Rayleigh fractionation line defined by modern Southern Ocean seawaters (Abouchami et al., 2011; Fig. 2d). If the Cd 
isotopic variations observed are controlled by changes in seawater salinity at the Xiaofenghe depositional site, estuarine mixing of seawater and river water, as described in Hohl et al. (2015), may account for the observed variation. The correlation between $\mathrm{Y} / \mathrm{Ho}$ and $\varepsilon^{112 / 110} \mathrm{Cd}$ (see Fig. $1 \mathrm{~g}$ and Fig. S-1c) may therefore be due to an increased riverine input, flattening the REE patterns, decreasing the total salinity and thus reducing the isotopic fractionation into calcite. Any further increase in riverine input would most likely drown out any potential biological signal in the stable isotope proxies, such as $\varepsilon^{112 / 110} \mathrm{Cd}$, which would then resemble typical crustal values.

\section{Implications}

Stable Cd isotopic compositions of Ediacaran-age carbonates from the Xiaofenghe section on the Yangtze Platform show significant variations of up to four $\varepsilon^{112 / 110} \mathrm{Cd}$ units. There are several possible reasons for these variations: part of the signal may be biological in origin while abiological (inorganic) processes almost certainly play a role as well. Factors controlling the Cd isotopic compositions may include fluid flow alteration, the precipitation of authigenic sulphides under anoxic pore water conditions, both processes possibly modifying $\varepsilon^{112 / 110} \mathrm{Cd}$ in the cap dolomites; additionally there are changes in $\mathrm{Cd}$ isotope fractionation into calcite as a function of salinity, as suggested here for the upper Doushantuo. Encouragingly, our salinity-corrected palaeo-seawater $\varepsilon^{112 / 110} \mathrm{Cd}_{\mathrm{sw}}$ signals overlap those of modern surface waters. A positive correlation between $\delta^{13} \mathrm{C}_{\text {carb }}$ and $\varepsilon^{112 / 110} \mathrm{Cd}$ is observed, which suggests that the $\mathrm{Cd}$ isotope fractionation might, in part, have a biological origin, meaning that phytoplankton were present as early as the Ediacaran. Future research on marine sediments will need to address the abiotic fractionation processes above, and correct for them, in order to arrive at any biologically-derived Cd isotope signal present.

\section{Acknowledgements}

This work was funded as part of DFG Research Group FOR-736 "The Precambrian-Cambrian Biosphere Revolution" (Subproject Be 1820/4-2). We thank Reimund Jotter and Wafa Abouchami at MPIC for discussions and their support in the lab, and members of FOR-736 who helped in sampling. We acknowledge helpful comments by Michael Tatzel, two anonymous reviewers and the editor, Helen Williams.

Editor: Helen Williams

\section{Additional Information}

Supplementary Information accompanies this letter at www.geochemicalperspectivesletters.org/article1704

Reprints and permission information is available online at http://www. geochemicalperspectivesletters.org/copyright-and-permissions

Cite this letter as: Hohl, S.V., Galer, S.J.G., Gamper, A., Becker, H. (2017) Cadmium isotope variations in Neoproterozoic carbonates - A tracer of biologic production? Geochem. Persp. Let. 3, 32-44.

\section{References}

Abouchami, W., Galer, S.J.G., de BaAr, H.J.W., Alderkamp, A.C., Middag, R., LaAn, P., Feldmann, H., ANDREAE, M.O. (2011) Modulation of the Southern Ocean cadmium isotope signature by ocean circulation and primary productivity. Earth and Planetary Science Letters 305, 83-91.

Ader, M., Sansjofre, P., Halverson, G.P., Busigny, V., Trindade, R.I.F., Kunzmann, M, NogUEIRA, A.C.R. (2014) Ocean redox structure across the Late Neoproterozoic Oxygenation Event: A nitrogen isotope perspective. Earth and Planetary Science Letters 396, 1-13.

BAU, M., Dulski, P., MölleR, P. (1995) Yttrium and holmium in South Pacific seawater: vertical distribution and possible fractionation mechanisms. Oceanographic Literature Review 42, 955.

BEBout, G.E., FogeL, M.L. (1992) Nitrogen-isotope compositions of metasedimentary rocks in the Catalina Schist, California: Implications for metamorphic devolatilization history. Geochimica et Cosmochimica Acta 56, 2839-2849.

Boyle, E.A., SCLATER, F., EDMOND, J.M. (1976) On the marine geochemistry of cadmium. Nature $263,42-44$.

BRAND, U., VEIZER, J. (1980) Chemical diagenesis of a multicomponent carbonate system-1: trace elements. Journal of Sedimentary Petrology 50, 1219-1236.

CANFIELD, D.E., GLAZER, A.N., FALKOWSKI, P.G. (2010) The evolution and future of earth's nitrogen cycle. Science 330, 192-196.

FRAMSON, P.E., LECKIE, J.O. (1978) Limits of coprecipitation of cadmium and ferrous sulfides. Environmental Science and Technology 12, 465-469.

Georgiev, S.V., Horner, T.J., Stein, H.J., Hannah, J.L., Bingen, B., RehKämper, M. (2015) Cadmium isotopic evidence for increasing primary productivity during the Late Permian anoxic event. Earth and Planetary Science Letters 410, 84-96.

HALVERSON, G.P., HURTGEN, M.T. (2007) Ediacaran growth of the marine sulfate reservoir. Earth and Planetary Science Letters 263, 32-44.

Horner, TJ SCHÖNB̈̈CHLER, M. REHKäMper, M, Nielsen, S.G, Wiluiams, H, HALliday, A.N., XUE, Z., HeIN, J.R. (2010) Ferromanganese crusts as archives of deep water Cd isotope compositions. Geochemistry, Geophysics, Geosystems 11, 1-10

Horner, T.J., RickABY, R.E.M., HENDERSON G.M. (2011) Isotopic fractionation of cadmium into calcite. Earth and Planetary Science Letters 312, 243-253.

Hohl, S.V., Becker, H., Gamper, A., Jiang, S.-Y., Wiechert, U., Yang, J.-H., WeI, H.-Z (2015) Secular changes of water chemistry in shallow-water Ediacaran ocean: Evidence from carbonates at Xiaofenghe, Three Gorges area, Yangtze Platform, South China. Precambrian Research 270, 50-79. 
IshiKaWA, T., Ueno, Y., SHU, D., LI, Y., HAN, J., GuO, J. (2013) The $\delta^{13} C$ excursions spanning the Cambrian explosion to the Canglangpuian mass extinction in the Three Gorges area, South China. Gondwana Research 25, 1045-1056.

Kaufman, A.J., KNOLL, A.H. (1995) Neoproterozoic variations in the C-isotopic composition of seawater: stratigraphic and biogeochemical implications. Precambrian Research 73, 27-49.

KNolL, A.H. (2015) Life on a Young Planet: The First Three Billion Years of Evolution on Earth Princeton University Press

LACAN, F., FrancoIS, R., JI, Y., SHeRreL, R.M. (2006) Cadmium isotopic composition in the ocean. Geochimica et Cosmochimica Acta 70, 5104-5118.

LAWRENCE, M.G., KAMBER, B.S. (2006) The behaviour of the rare earth elements during estuarine mixing-revisited. Marine Chemistry 100, 147-161.

LEWIS, B.L., LANDING, W.M. (1992) The investigation of dissolved and suspended-particulate trace metal fractionation in the Black Sea. Marine Chemistry 40, 105-141.

PARK, H., SONG, B., MOREL, F.M.M. (2007) Diversity of the cadmium-containing carbonic anhydrase in marine diatoms and natural waters. Environmental Microbiology 9, 403-413.

Planavsky, N., BekKer, A., Rouxel, O.J., Kamber, B., Hofmann, A., Knudsen, A., Lyons, T.W. (2010) Rare Earth Element and yttrium compositions of Archean and Paleoproterozoic Fe formations revisited: New perspectives on the significance and mechanisms of deposition. Geochimica et Cosmochimica Acta 74, 6387-6405.

PrICE, N.M., MoreL, F.M.M. (1990) Cadmium and cobalt substitution for zinc in a marine diatom. Nature 344, 658-660.

RipPeRgeR, S., ReHKÄMPER, M., PORCELlI, D. (2007) Cadmium isotope fractionation in seawater signature of biological activity. Earth and Planetary Science Letters 261, 670-684.

Rolison, J.M., Stirling, C.H., George, E., MiddaG, R., Gault-Ringold, M., Rijkenberg, M.J.A., DE BAAR, H.J.W. (2015) Biogeochemical cycling of the uranium, iron and cadmium isotope systems during oceanic anoxia: A case study of the Black Sea. Goldschmidt Abstracts 2015, 2671.

SACHS, J.P., REPETA, D.J. (1999) Oligotrophy and Nitrogen Fixation During Eastern Mediterranean Sapropel Events. Science 286, 2845-2488.

SchmitT, A.-D., GALER, S.J.G., AbouchamI, W. (2009) Mass-dependent cadmium isotopic variations in nature with emphasis on the marine environment. Earth and Planetary Science Letters 277, 262-272.

Struck, U., Pollehne, F., Bauerfeind, E., Bodungen, von B. (2004) Sources of nitrogen for the vertical particle flux in the Gotland Sea (Baltic Proper) - results from sediment trap studies.
Journal of Marine Systems 45, 91-101.

ThOMson, J., HigGS, N.C., JARVIS, I., Hydes, D.J. (1986) The behaviour of manganese in Atlantic carbonate sediments. Geochimica et Cosmochimica Acta 50, 1807-1818.

WASYLENKI, L.E., SWIHART, J.W., RomanielLo, S.J. (2014) Cadmium isotope fractionation during adsorption to Mn oxyhydroxide at low and high ionic strength. Geochimica et Cosmochimica Acta 140, 212-226.

Wen, H., Zhu, C., Zhang, Y., Cloquet, C., Fan, H., Fu, S. (2016) Zn/Cd ratios and cadmium isotope evidence for the classification of lead-zinc deposits. Scientific Reports 6, 25273, doi: 10.1038 /srep25273.

XiaO, S., McFadden, K.A., Peek, S., Kaufman, A.J., Zhou, C., Jiang, G., Hu, J. (2012) Integrated chemostratigraphy of the Doushantuo Formation at the northern Xiaofenghe section (Yangtze Gorges, South China) and its implication for Ediacaran stratigraphic correlation an .

YANG, J., LI, Y., LiU, S., TiAn, H., CHEN, C., LIU., J., SHI, Y. (2015) Theoretical calculations of Cd isotope fractionation in hydrothermal fluids. Chemical Geology 391, 74-82.

YIN, L., ZHU, M., KNOLL, A.H., YuAN, X., ZHANG, J., Hu, J. (2007) Doushantuo embryos preserved inside diapause egg cysts. Nature 446, 661-663. 